\title{
Der Platz der wissenschaftlich-technologischen Beziehungen im Prozeß der Europäischen Integration. Das Beispiel der Farbfernsehnorm
}

\author{
Ulrich PFEIL
}

Die Auswirkungen des technologischen Fortschritts auf die Entwicklung der internationalen Beziehungen hat in der Vergangenheit nur wenig Beachtung unter den Historikern hervorgerufen. ${ }^{1}$ Gleiches gilt für den Platz der wissenschaftlich-technologischen Beziehungen im Rahmen der europäischen Integration. Die Auseinandersetzung um die Einführung einer einheitlichen europäischen Farbfernsehnorm war in dieser Hinsicht sicherlich immer eine Ausnahme, ${ }^{2}$ kristallisierten sich in ihr doch nicht nur Nationalstolz und kulturpolitischer Missionsdrang, ${ }^{3}$ sondern zugleich «verband sich eine eigentlich rein technische Frage ab 1965 mit dem allgemeinen politisch-ideologischen Wettkampf der Blöcke NATO und Warschauer Pakt». ${ }^{4}$ Aus deutscher Sicht wird die Konfrontation zwischen dem französischen SECAM-(Séquentiel Couleur à Mémoire) und dem bundesdeutschen PAL-System (Phase Alternation Line) ${ }^{5}$ in erster Linie mit der sich vertiefenden Teilung Deutschlands in Zusammenhang gebracht, stand am Ende dieses technischen und politischen Wettbewerbs doch nicht mehr nur die Mauer zwischen beiden deutschen Staaten, sondern auch eine unterschiedliche Farbfernsehnorm. In diesem Beitrag wollen wir das Augenmerk jedoch auf die westeuropäischen Staaten als Schauplatz der deutsch-französischen Konkurrenz lenken, um den Platz der wissenschaftlich-technologischen Beziehungen im Prozeß der europäischen Integration zu beleuchten. Nach einem kurzen Überblick über den Platz der wissenschaftlich-technologischen Beziehungen im Rahmen der bundesdeutschen Außenpolitik ab Mitte der 1960er Jahre wollen wir uns auf Italien als Gründungsmitglied der

1. Vgl. U. TROITZSCH, Technikgeschichte, in: H.-J. GOERTZ (Hrsg.), Geschichte. Ein Grundkurs, Rowohlt, Reinbek, 22001, S.379-393.

2. Vgl. G. GLAUBITZ, Die PAL-SECAM-Kontroverse in der DDR. Die politisch-ideologische Instrumentalisierung der Farbfernsehfrage durch den ostdeutschen Staat zwischen 1965 und 1969, Aachener Beiträge zur Wissenschafts- und Technikgeschichte des 20. Jahrhunderts, Bd.5, gnt-Verlag, Diepholz, 2003.

3. Vgl. U. PFEIL, Die „anderen” deutsch-französischen Beziehungen. Die DDR und Frankreich 1949-1990, Böhlau, Köln, 2004; ders., Von der Politisierung eines technischen Wettstreits in den deutsch-französischen Beziehungen: Die PAL-SECAM-Kontroverse, in: Revue d'Allemagne et des pays de langue allemande, 1(2005), S.45-64.

4. G. GLAUBNITZ, PAL oder SECAM? Die ideologisch-politische Auseinandersetzung um das Farbfernsehsystem der DDR, in: Rundfunk und Geschichte. Mitteilungen des Studienkreises Rundfunk und Geschichte, Informationen aus dem Deutschen Rundfunkarchiv, 3/4(2003), S.132-137, hier S. 132.

5. Vgl. A.FICKERS, «Politique de la grandeur»versus «Made in Germany». Die Analyse der PAL-SECAM-Kontroverse als Beispiel einer politischen Kulturgeschichte der Technik, in: Nachrichtenblatt der deutschen Gesellschaft für Geschichte der Medizin, Naturwissenschaft und Technik, 3 (2003), S.169-180. 
EWG und Spanien als potentielles neues Mitglied konzentrieren, die ab Ende der 1960er Jahre zu einem wichtigen Aktionsfeld in dem Wettstreit um die Farbfernsehnorm wurden.

\section{Unterschiedliche Vorstellungen von Europa}

Seit den 1950er Jahren arbeiteten Techniker in Frankreich und der Bundesrepublik an der Entwicklung einer Farbfernsehnorm. Ein erstes brauchbares Ergebnis konnte der französische Ingenieur Henri de France (1911-1986) von der Compagnie Française de Télévision (CFT) vorlegen. Das von ihm entwickelte SECAM-System fand anfangs auch bei der deutschen Firma Telefunken Anklang, doch als der Telefunken-Ingenieur Walther Bruch (1908-1990) Anfang der 1960er Jahre sein viel stärker an die amerikanische Norm NTSC (National Television System Committee) angelehntes PAL-System präsentierte und die technischen Direktoren der ARD von der Überlegenheit seines Verfahrens im Jahre 1962 überzeugen konnte, entschieden sich die deutsche Industrie, die Bundespost und die westdeutschen Rundfunkanstalten für das PAL-Verfahren, das Bruch bis 1965 zur Marktreife gebracht hatte. ${ }^{6}$

Die Bundesregierung hatte in dieser Anfangsphase bei keinem der drei konkurrierenden Systeme einen überragenden technischen Vorteil ausgemacht, ging infolge der großen Kapitaldeckung des amerikanischen Systems und seiner bisher in der Welt erreichten Verbreitung jedoch davon aus, daß es sich durchsetzen werde, wie der Staatssekretär Karl-Günter von Hase dem französischen Informationsminister Alain Peyrefitte in einem Gespräch am 19. Januar 1965 mitteilte. Er schlug deshalb seinerseits vor, daß die Bundesrepublik und Frankreich noch einmal den gemeinsamen Versuch unternehmen sollten, die Kompatibilität der beiden Systeme zu erreichen. Auch Bundeskanzler Ludwig Erhard brachte in seinem Gespräch mit Charles de Gaulle am folgenden Tag noch das Farbfernsehen als «Feld der europäischen Kooperation» ${ }^{7}$ ins Spiel, doch begannen sich die Fronten zwischen den beiden Ländern in diesen Wochen endgültig zu verhärten.

Zwar wurde bei den fernsehtechnischen Tagungen in Wien (24. März bis 7. April 1965) und Oslo (22. Juni bis 22. Juli 1966) nochmals versucht, ${ }^{8}$ eine europäische Einigung zu erzielen, doch die zunehmende Politisierung der Farbfernseh-

6. Vgl. A.FICKERS, Coopération - confrontation - cohabitation. Les relations entre CSF/CFT et AEG-Telefunken en matière de brevets et licences de télévision en couleurs dans les années soixante, in: Revue d'Allemagne et des pays de langue allemande, 1 (2005), S.29-43.

7. Gespräch des Bundeskanzlers Erhard mit Staatspräsident de Gaulle in Rambouillet, 20.01.1965, in: AAPD 1965I, Dok. 26, S.149.

8. Vgl. A.FICKERS, Standardization as an Object of Systematical Technical Historiography - The case of Colour Television, in: W. HESSER (Hrsg.), Proceedings of the Second Interdisciplinary Workshop on Standardization Research, Hochschule der Bundeswehr, Hamburg, 1999, S.118141. 
normen führte schließlich zum Scheitern der Verhandlungen. Die bundesdeutsche Seite hatte in dieser Phase in erster Linie auf ökonomische Gewinnerwartungen und die eigene wirtschaftliche Potenz gesetzt, während das französische SECAM-System umgehend die Unterstützung durch die höchsten politische Kreise erhalten hatte. Diese Haltung war zum einen Ausdruck für die enge Verzahnung von Politik und Wirtschaft - André Malraux hatte in den 1960er Jahren wiederholt bekannt, daß das Fernsehen seiner Meinung nach im Dienste des Staates stehen müsse ${ }^{9}-$, zum anderen aber auch für das gaullistische Selbstverständnis, hatte der General doch selber den Satz geprägt «Qui tient la télévision tient un pays»! ${ }^{10}$ Für den stets um den «Rang» Frankreichs in der Welt besorgten Staatspräsidenten war das Fernsehen zugleich die Visitenkarte Frankreichs, wie Andreas Fickers konstatiert: «Dieser nationale Champion der Technik sollte der Welt die industrielle Potenz Frankreichs und damit die Erfolge der französischen Modernisierungsanstrengungen vor Augen führen». ${ }^{11}$ Das SECAM-System reihte sich damit in andere «eigenständige, von der amerikanischen Wirtschaft unabhängige Entwicklungen in den Zukunftstechnologien» ein, die «der französischen Emanzipation von der amerikanischen Hegemonie» dienten. ${ }^{12}$

Die Bundesregierung gedachte anfangs «eine technische Diskussion über die Qualitäten der verschiedenen Systeme nicht durch politisches Einwirken zu beeinflussen» ${ }^{13}$ und setzte ganz auf die weltweite Reputation von Made in Germany. Die Tendenz der französischen Regierung, «die Ausbreitung des französischen Systems mit politischen Mitteln» zu fördern, ${ }^{14}$ verärgerte die Bonner Politiker jedoch zunehmend. Sie konnten sich zudem dem Eindruck nicht verwehren, daß die von Frankreich angebotene Kooperation selbstredend der Übernahme des französischen SECAM-Systems gleichkomme. Die Farbfernsehnorm war für Paris in der Tat immer ein Mittel, um die politische Führungsrolle in Europa zu untermauern, das Paris als eigenständige Einheit nicht zuletzt in Abgrenzung zu den USA definierte. So wie de Gaulle in den Élysée-Vertrag eine antiamerikanische Komponente implantieren wollte, die dann durch die Präambel des Bundestages zunichte gemacht wurde, ${ }^{15}$ kultivierte Paris auch in der Farbfernsehfrage diese Argumenta-

9. Vgl. J.-N. JEANNENEY, Les médias, in: R. RÉMOND (Hrsg.), Pour une histoire politique, Seuil, Paris, 1988, S. 185-198, hier S.190.

10. Zit. nach A. PEYREFITTE, C'était de Gaulle, vol.2, Fallois, Paris 1997, S.386.

11. A.FICKERS, «Politique de la grandeur» ..., op.cit., Anm.5, S.173.

12. W. LOTH, Helsinki, 1. August 1975. Entspannung und Abrüstung, dtv, München, 1998, S.118 f.

13. AA an alle diplomatischen und Handelsvertretungen der Bundesrepublik Deutschland, 28.02.1966; PA/AA, B2, Bd.132, B1.86-88.

14. Werner Nestel, Generaldirektor von AEG-Telefunken, unternahm im November 1966 eine Reise durch mehrere westeuropäische Staaten, um mit den interessierten Regierungen über die Einführung des PAL-Systems zu verhandeln. Bei dieser Gelegenheit teilte ihm sein norwegischer Gesprächspartner mit, daß die Franzosen ihre Zustimmung zur Vergabe eines mit dem NATO-Frühwarnsystem zusammenhängenden Auftrags an Norwegen davon abhängig mache, daß Norwegen das SECAM-System übernehme, was von norwegischer Seite aber abgelehnt wurde; Aufzeichnung der bundesdeutschen Botschaft in Paris, 16.11.1966; PA/AA, B2, Bd.132, B1.179.

15. Vgl. C. DEFRANCE, U. PFEIL (Hrsg.), Le Traité de l'Élysée et les relations franco-allemandes 1945-1963-2003, CNRS Editions, Paris 2005 (deutsche Version: Oldenbourg, München 2005). 
tion und präsentierte eine deutsch-französische Gemeinschaftsproduktion als Gewinn für die europäische Sache, wie Peyrefitte bei den Regierungsbesprechungen vom 15. Februar 1964 betonte. ${ }^{16}$ Bis zu seinem Rücktritt blieb diese Angelegenheit daher für de Gaulle eine Prestigefrage, um den eigenen politischen Einfluß bzw. das «rayonnement de la France» sowohl jenseits des Eisernen Vorhangs wie auch in anderen westlichen europäischen Staaten zur Geltung zu bringen («Vom Atlantik bis zum Ural»). Bei dem «Atlantiker» Erhard verfingen antiamerikanische Argumente jedoch nicht, so daß auch die deutsch-französischen Regierungsgespräche vom Juli 1964 ohne konkrete Ergebnisse blieben ${ }^{17}$ und de Gaulle wie auch Peyrefitte resigniert feststellten:

«Solange der politische Wille jedoch dazu fehle, werde weder zwischen Deutschland und Frankreich ein gemeinsames Farbfernsehen zustande kommen, noch ein europäisches System verwirklicht werden». ${ }^{18}$

Daß sich Frankreich außenpolitisch nicht auf das westliche Lager bzw. auf die binären Grundstrukturen des Kalten Krieges beschränken wollte, ${ }^{19}$ hatte es bereits mit den französisch-sowjetischen Vereinbarungen über kulturellen Austausch (1963) und einen Handelsvertrag (1964) dokumentiert. Doch de Gaulle dachte nicht daran, auf halben Wege stehen zu bleiben, sondern förderte darüber hinaus die am 22. März 1965 erfolgte Unterzeichnung eines Farbfernsehabkommens, das die Übernahme des französischen SECAM-Systems für das sowjetische Farbfernsehen und eine wissenschaftliche und finanzielle Zusammenarbeit bei der Weiterentwicklung des SECAM-Verfahrens vorsah. ${ }^{20}$ Daß Frankreich die Bundesregierung entgegen den im Élysée-Vertrag fixierten deutsch-französischen Konsultationsmechanismen erst am 19. März 1965 darüber informierte, drei Tage später mit der UdSSR einen Vertrag über die Übernahme des SECAM-Systems unterschreiben zu wollen, ${ }^{21}$ bedeutete ein fait accompli und gehörte zu den kleinen Nicklichkeiten, die für die westdeutsch-französischen Beziehungen nach 1963 so typisch waren. De Gaulles am 8. Februar 1966 zum Ausdruck gebrachtes Bedauern, daß die Zusammenarbeit auf diesem Gebiet «nicht richtig geklappt habe» und es jetzt «natürlich zu spät» sei, «ein gemeinsames System zu errichten», 22 war dabei nicht mehr, als ein in diplomatische Formeln gewickelter Vorwurf an die bun-

16. Deutsch-französische Regierungsbesprechung in Paris, 15.02.1964, in: AAPD 1964II, Dok.50, S.254

17. Botschafter Berger, Den Haag, an das AA, 07.04.1965, in: AAPD 1965II, Dok.166, S.661, Anm.8.

18. Deutsch-französische Regierungsbesprechung in Bonn, 04.07.1964, in: AAPD 1964II, Dok.188, S.787.

19. Frankreich hatte die Bundesrepublik im Vorfeld der Vertragsunterzeichnung nicht konsultiert; vgl. Botschafter Berger, Den Haag, an das AA, 07.04.1965, in: AAPD 1965II, Dok.166, S.661.

20. Vgl. Europa-Archiv 1965, Z 73.

21. Vgl. W. KAISER, Die PAL-SECAM-Farbfernseh-Kontroverse, in: H. ALBRECHT (Hrsg.), Naturwissenschaft und Technik in der Geschichte. 25 Jahre Lehrstuhl für Geschichte der Naturwissenschaft und Technik am Historischen Institut der Universität Stuttgart, Verlag für Geschichte der Naturwissenschaften und Technik, Stuttgart, 1993, S.143-160, hier S.150.

22. Gespräche des Bundeskanzlers Erhard mit Staatspräsident de Gaulle in Paris, 08.02.1966, in: AAPD 1966I, Dok.36, S.175. 
desdeutsche Seite, die französischen Positionen nicht ausreichend unterstützt zu haben.

Schuldzuweisungen der französischen Seite wollte man im bundesdeutschen Außenministerium 1966 jedoch nicht gelten lassen, wo man darauf hinwies, daß sich das Bundesministerium für Post- und Fernmeldewesen erst 1964 «nach einer Verschärfung des deutsch-französischen Konkurrenzkampfes 1963/64» für das PAL-System entschieden habe:

\begin{abstract}
«Vorwürfe sind im Farbfernsehstreit fehl am Platz. Frankreich konnte 1963 aufgrund seiner langen Vorarbeiten [...] mit einer Übernahme von SECAM durch die Bundesrepublik rechnen. Wenn Telefunken 1964 ein mindestens ebenso gutes System vorstellte, das zudem eine enge Verbindung zu dem großen amerikanischen Markt ermöglichte, so war es legitim, daß die deutschen verantwortlichen Stellen diesem System den Vorzug gaben» ${ }^{23}$.
\end{abstract}

Als Frankreich 1966 schließlich das SECAM-Verfahren offiziell zur eigenen Farbfernsehnorm erklärte und die Bundesrepublik im folgenden Jahr das PAL-System auf der Internationalen Funkausstellung in Berlin als bundesdeutsche Fernsehnorm einführte, begann ein Wettbewerb, der auf politischer und technischer Ebene die Regeln des Fairplays immer wieder durchbrach. Obwohl der damalige Bundesaußenminister Willy Brandt an seinen französischen Amtskollegen Michel Debré in den Gesprächen vom 27. September 1968 erneut appelliert hatte, «über den französischen Interessen [...] die schwerwiegenden deutschen Interessen am Nichtzustandekommen dieses Geschäfts nicht zu vergessen», ${ }^{24}$ unterzeichneten Frankreich und die DDR am 4. März 1969 in Leipzig die Vereinbarung über die Zusammenarbeit auf dem Gebiet des Farbfernsehens, die zwar keinem völkerrechtlichen Vertrag gleichkam, doch an den «Wurzeln der Zusammengehörigkeit der beiden Teile Deutschlands» rührte. ${ }^{25}$ Die Zweiteilung des deutschen Farbfernsehhimmels besiegelten schließlich am 12. Mai 1969 die CFT, der VEB Fernsehgerätewerk Staßfurt und das Rundfunk- und Fernsehtechnische Zentralamt der Deutschen Post in einem Lizenzvertrag, ${ }^{26}$ so daß im Zuge des Starts des 2. Fernsehprogramms des Deutschen Fernsehfunks (DFF) am 3. Oktober 1969, wenige Tage vor dem 20. Jahrestag der DDR, das französische SECAM IIIb-Systems in der DDR zur offiziellen Farbfernsehnorm wurde.

23. Ausführungen des Vortragenden Legationsrats I. Klasse Steg, 28.02.1966, in: AAPD 1966I, Dok.52. S.238, Fußnote 5.

24. Vermerk des AA zur Reise des stellvertretenden «DDR»-Ministers Probst nach Frankreich, 30.10.1968; PA/AA, B 35, Bd.400.

25. Am 20.2.1969 hatte AA-Ministerialdirektor Frank Botschafter Freiherr von Braun angewiesen, de Gaulle auf diesen Befund bei dem am nächsten Tag angesetzten Frühstück hinzuweisen. Schließlich ergab sich für Braun aber nicht die Gelegenheit, die Angelegenheit gegenüber dem französischen Staatspräsidenten zur Sprache zu bringen; vgl. Aufzeichnung des Ministerialdirektors Frank, 27.02.1969, in: AAPD 1969I, Dok.83, S.294, Anm.6.

26. Vgl. Lizenzvertrag zwischen der Compagnie Française de Télévision und der VEB Fernsehgerätewerk Staßfurt und der Deutschen Post, Rundfunk- und Fernsehtechnisches Zentralamt, 12.05.1969; BAB, DL 2/VAN/904; Vertragsbericht 1968/69 - Frankreich; PA/AA, Bestand MfAA/C 1200/74, Bl.4f. 


\section{Die Politisierung der wissenschaftlich-technologischen Beziehungen}

Die deutsch-französische Konkurrenz in der Frage der Farbfernsehnorm hatte eine Entwicklung beschleunigt, der sich die Bonner Außenpolitik nun verstärkt stellte. Nachdem den technologisch-wissenschaftlichen Beziehungen in der Vergangenheit nur ein untergeordneter Platz eingeräumt worden war, betonte Außenminister Brandt in seiner vielbeachteten Rede aus Anlaß der Eröffnung der 25. Deutschen Funkausstellung in Berlin am 25. August 1967, daß Politik heutzutage «unter Außerachtlassung der Wirkungen des technologischen Fortschritts nicht mehr denkbar» sei, weil das wissenschaftlich-technische, das wirtschaftliche und das politische Potential der einzelnen Staaten in einer wechselseitigen Abhängigkeit zueinander stünden:

«Der schnelle Fortschritt von Wissenschaft und Technik, die über den nationalen Rahmen hinausreichenden Größenordnungen der modernen natur- und ingenieurwissenschaftlichen Forschung und die wachsende Erkenntnis der politischen Bedeutung von Forschung und Technologie haben die außenpolitische Bewertung des wissenschaftlich-technischen Entwicklungsstandes des eigenen Landes und anderer Staaten, die Beobachtung der Wissenschaftspolitik anderer Länder sowie die internationale Zusammenarbeit auf wissenschaftlich-technischem Gebiet zu einem neuen Bereich der Diplomatie und der internationalen Beziehungen werden lassen. Mehr und mehr Staaten bedienen sich des Instruments wissenschaftlich-technischer Zusammenarbeit, um politische Ziele zu erreichen».

Da Frankreich, die Sowjetunion und verschiedene kleine Staaten in der Vergangenheit unter Beweis gestellt hätten, daß wissenschaftlich-technologischen Beziehungen in hohem Maße eine politische Dimension zukomme, sprach sich auch Brandt dafür aus, daß dieser Bereich wichtigen politischen Zielen dienen könne wie der Friedensicherung, der Entspannung zwischen Ost und West, der Vertiefung der europäischen Integration und der Überwindung des Grabens zwischen den Industrie- und Entwicklungsländern. ${ }^{27}$ Diese Einsicht galt von nun an als Maxime bundesdeutscher Außenpolitik, wie aus einem Vermerk von 1970 hervorgeht: «Eine dynamische deutsche Außenpolitik verlangt auch zwingend eine ebenso dynamische auswärtige zwischenstaatliche Wissenschaftspolitik». ${ }^{28}$

Gerade in der Farbfernsehfrage ließen die Verantwortlichen in Bonn immer häufiger erkennen, daß auch sie auf diesem Feld nach politischen Kategorien zu handeln bereit waren, so daß sie die Entscheidungen dritter Staaten auf der politischen Soll- bzw. Habenseite verbuchten, wie im Fall Österreichs, dessen Entscheidung für das PAL-System Anfang 1967 von AA-Staatssekretär Rolf Lahr mit «Genugtuung» registriert wurde. ${ }^{29}$ Das Auswärtiges Amt stellte darüber hinaus Überlegun-

27. AA an alle deutschen diplomatischen und berufskonsularischen Vertretungen zur außenpolitischen Nutzung des deutschen wissenschaftlich-technischen Potentials, 29.01.1968; PA/AA, B 2, Bd.179, B1.156-162, hier: B1.161.

28. Vermerk über die Gedanken für die zukünftige Zusammenarbeit zwischen der Kulturabteilung des AA und dem Bundesministerium für Bildung und Wissenschaft auf dem Gebiet der Wissenschaft; 29.07.1970; PA/AA, ZA 106.179. 
gen an, ob es nicht zum einen aktiver in Ländern wie Großbritannien, Italien, den Niederlanden und skandinavischen Staaten werden solle, um das Erreichte zu verteidigen, zum anderen den Aktionsbereich in Griechenland, Spanien, Portugal, der Schweiz u.a. weiter verstärken müsse, um diese zu einer Entscheidung für das PAL-System zu bewegen. ${ }^{30}$ In diesen Überlegungen kam der wachsende Unwille der bundesdeutschen Seite zum Ausdruck, die französische Auffassung von der europäischen Einigung zu akzeptieren, die nach Meinung von AA-Ministerialdirektor Max Frank «einen Prozeß der Zuordnung der anderen Länder an Frankreich» vorsehe, was er auf die fehlenden französischen Erfahrungen mit dem Föderalismus zurückführte. ${ }^{31}$

Als Folge des deutsch-französischen Zerwürfnisses intervenierte die Bundesregierung nun immer öfter direkt bei Spitzengesprächen, so im Februar 1969, als Brandt seinen italienischen Amtskollegen Pietro Nenni bat, «Italien möge sich nicht aus politischen Gründen etwa für das technisch schwächere SECAM-System entscheiden, nachdem es sich von der Überlegenheit des PAL-Systems überzeugt habe». ${ }^{32}$ Nenni sprach in seiner Antwort von einer «Grundsatzentscheidung zugunsten des PAL-Systems», doch erschien die Frage angesichts des zunehmenden französischen Drucks wieder offener. Auf welche Weise Frankreich den Wettstreit für sich entscheiden wollte, verdeutlichte ein höherer Beamter aus dem italienischen Außenministerium am 26. Februar 1969 der bundesdeutschen Botschaft:

«Die Franzosen hätten dem Präsidenten der FIAT-Werke Agnelli, Konzessionen hinsichtlich einer größeren Aktienbeteiligung an Citroën in Aussicht gestellt, falls er sich für SECAM verwende. Darüber hinaus böten die Franzosen auch anderen unmittelbar interessierten Industriezweigen Konzessionen an». ${ }^{33}$

Trotz dieses «ungewöhnlichen Drucks» wollte die Bundesregierung an der Politisierungsspirale nicht in gleicher Weise mitdrehen, wie Staatssekretär Lahr gegenüber dem italienischen Botschafter in Rom unterstrich:

«Den Versuch, über die italienische Industrie eine Entscheidung der Regierung zu beeinflussen, hielten wir nicht für eine faire Methode. Auch wir hätten die Möglichkeit, solche Junktims herzustellen, möchten dies aber nicht tun». ${ }^{34}$

Frankreich erhöhte jedoch seine industriellen Aktivitäten und den politischen Druck in Italien gerade aus strategischen Gründen immer weiter:

«Es geht - sicherlich nicht zu unrecht - davon aus, daß, wenn Italien umgefallen ist, auch Spanien und Jugoslawien umfallen werden und daß damit eine weitere Kettenreaktion im Mittelmeerraum ausgelöst wird, die sogar nach Lateinamerika weiterrei-

29. Aufzeichnung des Ministerialdirigenten Frank von einem Gespräch zwischen St.S Rolf Lahr und dem österreichischen Außenminister Dr. Lujo Tonèiæ-Sorinj in Bonn, 28.02.1967, in: AAPD 1967I, Dok.76, S.77.

30. Aufzeichnung des DG I A zur Situation des Farbfernsehens, 18.10.1966; PA/AA, B 35, Bd.212.

31. Aufzeichnung des Ministerialdirektors Frank, 05.03.1969, in: AAPD 1969I, Dok.89, S.321.

32. Gespräch des Bundesministers Brandt mit dem italienischen Außenminister Nenni in Rom, 14.02.1969, in: AAPD 1969I, Dok.60, S.205.

33. AAPD 1969I, Dok.83, S.295, Anm.7.

34. Aufzeichnung des Ministerialdirektors Frank, 27.02.1969, in: AAPD 1969I, Dok.83, S.295. 
chen kann. Italien nimmt also für Frankreich - und natürlich auch für uns - eine Schlüsselposition ein». ${ }^{35}$

Die Situation schien sich im Frühjahr 1969 zuzuspitzen, sahen doch auch die bundesdeutschen Diplomaten nach Besprechungen mit italienischen Vertretern aus Politik und Industrie die Entscheidung wieder «auf des Messers Schneide», so daß sich Bonn die Frage stellte, ob man nicht selber die Politisierung vorantreiben solle. Das starke politische Engagement Frankreichs wurde bei manchen italienischen Verantwortlichen in Politik und Wirtschaft durchaus positiv aufgenommen und als allgemeines Interesse für den Nachbarn gewertet, während die stärkere politische Zurückhaltung der bundesdeutschen Seite mit Mißfallen beobachtet wurde und in die Aufforderung mündete, sich mehr anzustrengen:

«Hierbei wird unterstrichen, daß es dabei nicht nur um technisch-kommerzielle Fragen gehe, sondern der politische Aspekt sehr wichtig sei. Nachdem die Franzosen die Angelegenheit stark politisiert hatten, bliebe uns, wenn wir nicht aufgeben wollten, nichts anderes übrig, als diese Frage ebenfalls als eine politische zu sehen».

Da Frankreich seine industriellen, technischen und kommerziellen Beziehungen mit Italien allgemein ausbaute, um die Regierung in Rom doch noch zu einem Umdenken zu bewegen, wurde auch in Bonn angedacht, «in anderen technologischen Bereichen mit Italien stärker zusammenzuarbeiten». Das AA entschloß sich schließlich, sich «nicht mehr mit der Herausstellung der rein technisch-wirtschaftlichen Vorteile des PAL-Systems zu begnügen» und über eine breitere wissenschaftlich-technologische Zusammenarbeit nachzudenken:

«Vielmehr dürfte es angebracht sein, die deutschen Bemühungen um Einführung des PAL-Systems in anderen europäischen Ländern im Zusammenhang mit anderen Bereichen technologischer und industrieller Zusammenarbeit mit diesen Bereichen zu sehen, um geeignete Schritte zur Unterstützung der deutschen Bemühungen zur Einführung des PAL-Systems festlegen zu können». ${ }^{36}$

Wichtigstes Argument, um die Italiener zur Übernahme des PAL-Systems zu bewegen, blieb jedoch die europäische Integration, wie aus einem Gesprächsvorschlag für die Gespräche zwischen Brandt und seinem Amtskollegen Aldo Moro hervorgeht:

«Mit den PAL-Ländern der EWG und EFTA teilt Italien ein hohes Maß an Übereinstimmung über die Notwendigkeit der europäischen Integration und der westeuropäischen Zusammenarbeit. Wir sind davon überzeugt, daß die Einführung des PAL-Systems das Ziel, eine größere europäische Kohäsion herzustellen, fördern könnte». ${ }^{37}$

35. Botschafter Lahr, Rom, an das AA, 02.04.1969, in: AAPD 1969I, Dok.115, S.444.

36. AA an BM für das Post- und Fernmeldewesen, Presse- und Informationsamt der Bundesregierung, BM für Wirtschaft, BM für wissenschaftliche Forschung, 01.04.1969; PA/AA, B 35, Bd.401.

37. Gesprächsvorschläge für die Gespräche des Herrn Ministers mit dem italienischen Außenminister Moro am 1. September 1969, 21.08.1969; PA/AA, B 35, Bd.401. Auch für die Gespräche zwischen den beiden Außenministern am 01.12.1969 schlug die Abt. IA6 vor, den «Gesichtspunkt der europäischen Einheitlichkeit auf dem Gebiet des Farbfernsehens» ins Spiel zu bringen; Vorschläge für die Gesprächsführung zur wissenschaftlich-technologischen Zusammenarbeit, 28.11.1969; PA/AA, B 35, Bd.401. 
Weiterhin brachte das Auswärtige Amt in den Gesprächen mit der italienischen Regierung ins Spiel, daß mit der Entscheidung für das PAL-Verfahren die Gefahr gebannt sei, Mittel- bzw. Nordeuropa und der Mittelmeerraum könnten getrennt werden:

«Hierbei ist namentlich an Großbritannien zu denken. Die technologische Zusammenarbeit zwischen Großbritannien und den Ländern der Europäischen Gemeinschaft wird von der Mehrzahl dieser Länder nachdrücklich gefördert. Das Farbfernsehen bietet hierzu eine leicht realisierbare Gelegenheit auf einem Gebiet großer Zukunft und spezifischer politischer Bedeutung».38

Im April 1970 konnte Außenminister Walter Scheel gegenüber dem italienischen Ministerpräsidenten Mariano Rumor darauf verweisen, daß sich nach der positiven spanischen Entscheidung 14 europäische Länder für das PAL-System ausgesprochen hätten. ${ }^{39}$ Doch Italien kam nicht nur im südeuropäischen Raum eine Schlüsselposition zu, sondern im ganzen Mittelmeerbereich, insbesondere auch für die arabischen Staaten. In diesem Zusammenhang wies der nunmehrige Botschafter Rolf Lahr den italienischen Ministerpräsidenten Emilio Colombo im März 1971 darauf hin, daß das französische Argument, «eine einheitliche Fernsehpolitik auf der Basis von SECAM im Mittelmeerraum» zu schaffen «schon deswegen illusionär sei, weil die neben Italien und Frankreich wichtigsten Länder, Spanien, Jugoslawien, ${ }^{40}$ sich bereits für PAL entschieden hätten». ${ }^{41}$ Da Frankreich in der westlichen Hemisphäre immer stärker in das Hintertreffen geriet, konnte die Bundesregierung das PAL-System nicht unbegründet als europäische Lösung anpreisen, was sie weniger in den Verdacht brachte, nationale politische Interessen zu verfolgen, wie Botschafter Lahr nach Bonn meldete:

«Die im November von dem Herrn Bundeskanzler gewählte Formel >wir hoffen auf eine europäische Option Italiens<, ist m.E. die geeignetste. Sie hat seinerzeit ihren Eindruck nicht verfehlt». ${ }^{42}$

Gleichzeitig konnte es aber auch der bundesdeutschen Selbstdarstellung im Ausland und dem Beweis von der Leistungskraft der eigenen Wirtschaft und Forschung dienen, galt es im Ausland - wie das AA wußte - doch als «deutsches System» und wurde «nicht mit einer bestimmten Firma assoziiert». ${ }^{43}$ Die französische Regierung schien mit ihrer «insistente[n] Art» hingegen den Bogen überspannt und für Verstimmungen in Rom gesorgt zu haben, ${ }^{44}$ so daß sich Italien schließlich 1975 für das PAL-System entschied.

38. Gründe für die Einführung des PAL-Farbfernsehsystems in Italien, [o.D./September 1969]; PA/ AA, B 35, Bd.401.

39. Vgl. Gespräch des Bundesministers Scheel mit Ministerpräsident Rumor in Rom, 08.04.1970, in: AAPD 1970I, Dok.149, S.580.

40. Vgl. zur Bedeutung Jugoslawiens in dem deutsch-französischen Wettstreit: Botschaftsrat Loeck, Belgrad, an das AA, 23.11.1967, in: AAPD 1967III, Dok.398, S.1537-1539.

41. Botschafter Lahr, Rom, an das AA, 24.03.1971, in: AAPD 1971I, Dok.106, S.498.

42. Botschafter Lahr, Rom, an das AA, 24.03.1971, in: AAPD 1971I, Dok.106, S.498.

43. Vermerk der Abt. IA6 im AA zum PAL-Farbfernsehen in Italien, 29.06.1970; PA/AA, B 35, Bd.401. 


\section{Wissenschaftlich-technologische Beziehungen: Ein Instrument zur Vorbereitung der Erweiterung}

Ein weiteres wichtiges Aktionsfeld war aus vielerlei Gründen Spanien, das nicht nur im Mittelmeerbereich eine zentrale Rolle einnahm, sondern neben seinem Einfluß in Mittel- und Südamerika auch in gewisser Weise Sprungbrett nach Nordafrika war. Zudem gehörte es zu jenen Ländern, die sich um die Aufnahme in die Europäische Gemeinschaft bemühten. Madrid hatte für 1969 eine Entscheidung in der Frage der Farbfernsehnorm angekündigt, seinen Entschluß jedoch u.a. auch von Italien abhängig machen wollen. Die Bundesrepublik gedachte anfangs ihre bisherige Linie beizubehalten und vertrat auch im spanischen Fall die Auffassung, «daß die Entscheidung für die Einführung des Farbfernseh-Systems in erster Linie nach wirtschaftlichen und technischen Kriterien getroffen werden sollte». ${ }^{45}$ Doch schnell wurde sich Bonn bewußt, daß der Konkurrenzkampf zwischen PAL und SECAM auch in Spanien in erster Linie auf politischem Feld ausgefochten wurde, wie das AA mit Mißfallen feststellte:

«Wie auch in Italien ist nun ebenfalls in Spanien eine massive politische und wirtschaftliche Einflußnahme der französischen Regierung mit dem Ziel festzustellen, Spanien von seiner Haltung gegenüber dem PAL-System abzubringen und für das SECAM-System zu gewinnen». ${ }^{46}$

Zu einer ersten Kraftprobe kam es im März 1969, als der in Madrid stattfindende Grand Prix de l'Eurovision de la Chanson die deutsch-französische Konkurrenz ein weiteres Mal anheizte:

«Die französische ORTF hat bereits ihre Mithilfe, d.h. insbesondere die Zurverfügungstellung eines Ü-Wagens, angeboten. Das würde aber eine Aufnahme und Weiterleitung durch SECAM mit nachfolgender Transcodierung für alle PAL-Länder bedeuten. Gleichzeitig könnte dieses propagandistisch so ausgenutzt werden, als ob sich Spanien für SECAM entschieden habe. Um dies zu vermeiden, müßten die deutschen Fernsehgesellschaften einen PAL-Farbfernseh-Ü-Wagen zur Verfügung stellen».

Die bundesdeutsche Botschaft in Madrid schrieb:

«Die Botschaft hält es im Rahmen ihrer Bemühungen um enge Zusammenarbeit auf kulturellem, technologischem und wirtschaftlichem Gebiet für wünschenswert, daß hierfür ein deutscher Übertragungswagen zur Verfügung gestellt wird». ${ }^{47}$

44. Vgl. zu den verschiedenen Arten der Beeinflussung durch die französische Seite auf den unterschiedlichen Ebenen: Botschafter Lahr, Rom, an das AA, 27.05.1970, in: AAPD 1970II, Dok.239, S.878 f.; Botschafter Lahr, Rom, an Bundesminister Scheel, 11.06.1970, in: ebenda, Dok.265, S.973-975.

45. Vermerk der Abt. IA6 zum heutigen Essen mit einer spanischen Delegation auf dem Petersberg, 25.05.1969; PA/AA, B 35, Bd.398.

46. Aufzeichnung der Abt. I zum Farbfernsehen in Spanien, 31.03.1969; PA/AA, B 35, Bd.398.

47. Bundesdeutsche Botschaft in Madrid an AA, 04.02.1969; PA/AA, B 35, Bd.400. Der WDR erklärte daraufhin seine Bereitschaft, einen Ü-Wagen zu schicken; Diplogerma der Bundesdeutschen Botschaft in Madrid an AA, 04.02.1969; PA/AA, B 35, Bd.400. 
Wie hart die Konkurrenz zwischen der Bundesrepublik und Frankreich gerade in Spanien war, unterstreicht das Angebot des französischen Industrieministers André Bettencourt, der der Regierung in Madrid für den Fall einer Übernahme des SECAM-Systems folgendes offerierte: 1) Finanzierungshilfe auf zehn Jahre; 2) technische Unterstützung im Farbfernsehbereich; 3) technische Unterstützung für den gesamten Elektronikbereich. ${ }^{48}$ Gleichzeitig habe die französische Seite nach bundesdeutschen Informationen politische Gesichtspunkte geltend gemacht, «insbesondere das Nachbarschaftsverhältnis zu Frankreich und das angebliche Interesse anderer Mittelmeerländer, vor allem Italiens, an der Einführung des SECAM-Systems». ${ }^{49}$

Genauso wie alle anderen EWG-Mitgliedstaaten konnte und wollte es sich die Bundesregierung nicht leisten, das francistische Spanien bei seinem Eintrittsgesuch voll zu unterstützen. Dagegen unterstützte Bonn den Madrider Wunsch, die Bedingungen des Präferenzabkommens zwischen Spanien und der EWG vom 1. Oktober $1970 \mathrm{zu}$ verbessern, da sich für Spanien durch den Eintritt von Großbritannien, Irland und Dänemark schwierigere Exportkonditionen abzeichneten. Franco wollte sich mit einem solchen Zugeständnis jedoch nicht begnügen, hatte er doch bereits 1962 einen Aufnahmeantrag in die EWG gestellt. Ein Druckmittel ergab sich aus der deutsch-französischen Konkurrenzsituation in der Farbfernsehfrage, konnte Madrid die beiden Wettbewerber doch immer wieder gegeneinander ausspielen.

Die Regierungen in Bonn und Paris mußten zudem auf den Druck aus dem eigenen Land reagieren, wollte sich doch weder die deutsche noch die französische Industrie Spanien als Absatzmarkt und Ort für langfristige, kapitalträchtige Investitionen entgehen lassen. Und in der Tat drängte AEG-Telefunken Bonn im Februar 1969 infolge der Interventionen der Regierung in Paris dazu, sich weiterhin auf politischer Ebene für das PAL-System einzusetzen. Nach einer Südamerikareise des Bundesaußenministers appellierte der stellvertretende Vorsitzende des Vorstandes der AEG, Felix Herriger, an Brandt, nach den Anfangserfolgen des PAL-Systems diesen auch Dauerhaftigkeit zu verleihen:

«Dies ist auch einhellig die Ansicht der mit diesem Thema befaßten politischen, wirtschaftlichen und für die Öffentlichkeitsarbeit zuständigen Sachverständigen der Bundesregierung sowie insbesondere auch der Auslandsvertretungen. Alle Stellen befürworten die Fortsetzung der Aktivität, hauptsächlich weil sie in der Zusammenarbeit auf dem Farbfernsehgebiet durch die Einführung des PAL ein gutes und in dieser Form selten wiederkehrendes Mittel sehen, das Ansehen der Bundesrepublik, ihrer Wirtschaft und Kultur [...] und damit die Beziehungen zu diesen Ländern zu fördern und zu festigen». ${ }^{50}$

48. Fernschreiben der bundesdeutschen Botschaft in Madrid an das AA, 25.03.1969; PA/AA, B 35, Bd.400.

49. Abt. IA6 (Günther Harkort) des AA an den Bundesminister für das Post- und Fernmeldewesen, Werner Dollinger, 02.04.1969; PA/AA, B 35, Bd.398.

50. Felix Herriger an Willy Brandt, 12.02.1969; B 35, Bd.154, 
Diesen Forderungen konnte sich das AA nicht völlig verwehren. So hieß es in einem Gesprächsvorschlag im Vorfeld eines Arbeitsessens mit einer spanischen Delegation im Mai 1969:

«Zahlreiche europäische Länder Europas haben sich bereits für das PAL-System entschieden; für das SECAM-System dagegen haben sich außer Frankreich, wo es seinen Ursprung hat, nur die Länder des Sowjetblocks entschieden». ${ }^{51}$

Gerade dieses Argument schien dem AA gegenüber dem francistischen Regime in Spanien aussichtsreich, wie es gegenüber der bundesdeutschen Botschaft in Madrid im Juli 1969 nochmals betonte: «Ein nicht zu unterschätzender Faktor dürfte die Tatsache sein, daß sich in Europa bisher für das SECAM-System außer Frankreich nur kommunistisch regierte Staaten entschieden haben».52 Als weiteres politisches Argument diente der bundesdeutsche Seite der südamerikanische Markt, wo sich das PAL-System in aussichtsreicher Konkurrenz zur amerikanischen Farbfernsehnorm NTSC befand und die Franzosen mit ihrem SECAM-System nicht angetreten waren: «Wegen seiner kulturellen Verbundenheit mit Lateinamerika dürfte es für Spanien auch aus diesem Grund vorteilhaft sein, sich für das PAL-System zu entscheiden». ${ }^{53}$ Spanien selber hatte zudem kommerzielles Interesse daran, daß sich Bonn auch für die Übernahme des PAL-Systems durch Marokko einsetze, wie der Botschaft Staatssekretär Günther Harkort zu verstehen gab:

«Das marokkanische Fernsehen übernehme viele spanische Programme. Wie er uns schon früher dargelegt hat, sei die spanische Regierung sehr daran interessiert, das marokkanische Fernsehen auch nach der Einführung des PAL-Systems mit Programmen zu versorgen». ${ }^{54}$

Dieses Anliegen wiederholte Botschafter José de Erice auch nach der offiziellen positiven Entscheidung seiner Regierung für das PAL-System, indem er hoffte, «daß dieser Beschluß der spanischen Regierung auch günstige Auswirkungen auf andere Länder des Mittelmeeres haben wird, mit denen Spanien eng und brüderlich verbunden ist». ${ }^{55}$

Nachdem sich die Regierung in Madrid am 24. Oktober 1969 für das PAL-System entschieden hatte, teilte der spanische Botschafter der Bundesregierung diesen Beschluß einen Tag später mit, ${ }^{56}$ was der neue Bundesaußenminister Walter Scheel in einem Gespräch mit seinem spanischen Amtskollegen dann auch mit Befriedigung kommentierte, «da auf diese Weise die Übertragung der Fernsehprogramme innerhalb Europas erleichtert würde». ${ }^{57}$ Staatssekretär Harkort dankte sei-

51. Vermerk der Abt. IA6 zum heutigen Essen mit einer spanischen Delegation auf dem Petersberg, 25.05.1969; PA/AA, B 35, Bd.398.

52. Abt. IA6 im AA an die bundesdeutsche Botschaft in Madrid, 07.07.1969; PA/AA, B 35, Bd.398.

53. Vermerk der Abt. IA6 zum heutigen Essen mit einer spanischen Delegation auf dem Petersberg, 25.05.1969; PA/AA, B 35, Bd.398.

54. Vermerk von Staatssekretär Harkort, 23.10.1969; PA/AA, B 35, Bd.398.

55. Jose de Erice an Harkort, 25.10.1969; PA/AA, B 35, Bd.398.

56. Aufzeichnung des Ministerialdirektors Herbst, 26.01.1970, in: AAPD 1970I, Dok.24, Anm.3, S.90. 
nerseits dem spanischen Botschafter, habe sein Land doch im Mittelmeerraum ein Signal gesetzt, «das, so hoffe ich mit Ihnen, seine Wirkung auf andere Länder jener Region haben wird». ${ }^{8}$ Jedes weitere Land, das sich für das PAL-System entschied, erleichterte Bonn eine europäische Argumentation, doch auch die spanische Presse reagierte in ähnlicher Weise nach der offiziellen Entscheidung der Regierung in Madrid, indem sie darauf hinwies, daß ausschlaggebend u.a. gewesen sei, ein mit den meisten anderen europäischen Ländern übereinstimmendes Fernsehsystem zu haben, was nicht zuletzt die Zusammenarbeit in der Eurovision erleichtere. ${ }^{59}$ Noch ein Jahr später war es dem AA-Ministerialdirigenten Klaus Simon wichtig zu betonen, daß sich die spanische Regierung nicht zuletzt auch zugunsten der Bundesrepublik entschieden habe, weil die Bonner Diplomatie von gleichberechtigten wechselseitigen Interessen im bilateralen Verhältnis zwischen beiden Ländern ausgegangen sei. ${ }^{60}$

Ausschlaggebend für die positive Entscheidung in Madrid war in der Tat, ${ }^{61}$ daß die neue sozial-liberale Koalition unter Bundeskanzler Willy Brandt die wissenschaftlich-technologischen Beziehungen mit einer Entwicklungspolitik koppelte, die den Anspruch hatte frei «außenpolitischer und wirtschaftspolitischer >Bevormundung «» zu sein, ${ }^{62}$ zugleich aber auch die eigenen wirtschaftlichen Ziele nicht aus den Augen verlor. Bereits Anfang 1969 hatte die bundesdeutsche Seite den Spaniern den Entwurf eines wissenschaftlich-technologischen Abkommens vorgeschlagen, das sie jedoch implizit von der Übernahme des PAL-Systems abhängig machte:

«Es wäre ein schlechter Beginn für ein derartig wichtiges deutsch-spanisches Abkommen, wenn ungefähr gleichzeitig nicht das technisch und wirtschaftlich höherwertige deutsche PAL-System, sondern das weniger ausgereifte französische SECAM-System in Spanien eingeführt würde». ${ }^{63}$

Konkret handelte es sich um die Umleitung eines Teils des Wassers des Tajo in den Segura, um das hydrographische Gleichgewicht zwischen den spanischen Provinzen zu verbessern. Dieses Projekt war gerade für die weitere wirtschaftliche Entwicklung der trockenen Gegenden im Südosten Spaniens von großer Bedeutung und sollte die Lebensbedingungen der dortigen Bevölkerung entscheidend verbessern. ${ }^{64}$ Dieses Bewässerungsprojekt ${ }^{65}$ wurde auch von spanischer Seite in die Ver-

57. Vgl. die Einzelheiten in: Gespräch des Bundesministers Scheel mit dem spanischen Außenminister Gregorio López Bravo in Madrid, 22.04.1970, in: AAPD 1970I, Dok.172, S.640 f.

58. Harkort an de Erice, 31.10.1969; PA/AA, B 35, Bd.398.

59. Vgl. Fernschreiben der bundesdeutschen Botschaft zu den Reaktionen der spanischen Presse, 27.10.1969; PA/AA, B 35, Bd.398.

60. Aufzeichnung des Ministerialdirigenten Simon, 30.08.1971, in: AAPD 1971II, Dok.286, S.1308.

61. «Dies gilt z.B. im Augenblick für Spanien, dem wir nicht zuletzt mit Rücksicht auf die für uns in vielfältiger Hinsicht wertvolle Entscheidung Madrids zugunsten des deutschen PAL-Systems für ein wirtschafts- und sozialpolitisch sinnvolles Entwicklungsprojekt eine im übrigen bescheidene Kapitalhilfe zukommen lassen wollen»; ebenda.

62. Aufzeichnung des Ministerialdirektors Herbst, 26.01.1970, in: AAPD 1970I, Dok.24, S.90.

63. Aufzeichnung der Abt. I zum Farbfernsehen in Spanien, 31.03.1969; PA/AA, B 35, Bd.398.

64. Vermerk der Abt. IA6 zum Tajo-Segura-Projekt, 24.06.1970; PA/AA, B 35, Bd.401.

65. Aufzeichnung der Abt. I über ein Gespräch von Staatssekretär Harkort mit dem spanischen Botschafter, 02.04.1969; PA/AA, B 35, Bd.398. 
handlungen um die Farbfernsehnorm eingebracht, wie der spanische Botschafter gegenüber seinen bundesdeutschen Gesprächspartnern deutlich machte: «Eine kooperative Haltung der Bundesregierung in der Frage Tajo-Segura würde die Übernahme des deutschen Farbfernseh-Systems PAL durch Spanien erleichtern» ${ }^{66}$. $\mathrm{Zu}$ einem Abkommen zwischen beiden Ländern kam es am 24. Juni 1970, in dem die bundesdeutsche Seite sich zu einer Beteiligung an der Finanzierung verpflichtete. In dem Schreiben an seinen spanischen Amtskollegen unterstrich BundesauBenminister Walter Scheel den Zusammenhang zwischen den beiden Projekten:

«Das Abkommen und die Entscheidung Ihrer Regierung zugunsten des PAL-Farbfernsehsystems sind weitere Beweise der guten und vertrauensvollen Zusammenarbeit zwischen unseren beiden Ländern im technologischen Bereich mit darüber hinausreichenden Auswirkungen». ${ }^{67}$

Richten wir abschließend unseren Blick nur auf die westlichen Länder, dann können wir zweifellos feststellen, daß die bundesdeutsche Farbfernsehnorm siegreich aus diesem deutsch-französischen Wettstreit hervorgegangen war, ${ }^{68}$ gelang es Frankreich doch nicht, sein SECAM-System in den EWG-Ländern durchzusetzen ${ }^{69}$ das für den nach Prestige und «Rang» strebenden französischen Staatspräsidenten eine erneute Gelegenheit sein sollte, sich als bedeutende Wissenschaftsnation zu profilieren und sich damit der Augenhöhe der beiden Großmächte anzunähern, auch wenn das fehlende wirtschaftliche Potential diese Bemühungen zu einem Drahtseilakt ohne Netz machte. Die Bundesregierung hatte anfänglich nicht die Absicht, diese Konkurrenz auf politischer Ebene zu führen, mußte jedoch erkennen, daß die wissenschaftlich-technologischen Beziehungen zu einem integralen Element staatlicher Außenpolitik avanciert waren. Ihre politische Dimension wurde den Deutschen in aller Deutlichkeit vor Augen geführt, als das französische System in der DDR eingeführt wurde, so daß es in beiden deutschen Staaten keine einheitliche Farbfernsehnorm mehr gab.

So standen schließlich beide Länder am Ende als Verlierer da, was Albert Probst, ehemaliger parlamentarischer Staatssekretär beim Bundesminister für Forschung und Technologie (1982-1991), zu der Schlußfolgerung führte, daß es nicht im gemeinsamen Interesse beider Länder liege, «sich bei derartigen Entwicklungen auf dem Weltmarkt Konkurrenz zu machena». ${ }^{70}$ Solange es aber de Gaulles Ziel war, ein «europäisches Europa» zwischen den beiden antagonistischen Großmächten aufzubauen, das Frankreichs Interessen zu dienen habe und in dem es nach dem

66. Aufzeichnung des Leiters der Abt. III im AA zum spanischen Bewässerungsvorhaben Tajo-Segura, 25.07.1969; PA/AA, B 35, Bd.398.

67. Scheel an Gregorio Lopez Bravo, 09.07.1970; PA/AA, B 35, Bd.401.

68. 1979 betrug die Zahl der PAL-Länder 60, das NTSC-System kam auf 28, das SECAM-System auf 24 Länder.

69. Vgl. A. FICKERS, PAL/SECAM: «Politique de la grandeur» contre «Made in Gernany», in: R. BELOT, u.a. (Hrsg.), La technologie au risque de l'Histoire, Berg International, Paris, 2000, S.343-353.

70. A. PROBST, Eröffnungsansprache, in: Y. COHEN, K. MANFRESS (Hrsg.), Frankreich und Deutschland. Forschung, Technologie und industrielle Entwicklung im 19. und 20. Jahrhundert, Beck, München, 1990, S. XXI. 
Selbstverständnis des Generals «unbestrittener Primus» ${ }^{71}$ sein sollte, blieb die Kooperation in Europa ein schwieriges Unterfangen, wie nicht zuletzt auch die «Krise des leeren Stuhls» (1965/66) zeigte, in der Frankreich die Sitzungen des Ministerrats boykottierte, um gegen die bevor stehende Einführung der qualifizierten Mehrheit als Abstimmungsprinzip im Rat zu demonstrieren. Als Lernprozeß kann es daher verstanden werden, daß sich Frankreich und die Bundesrepublik Ende der 1960er Jahre, als der PAL-SECAM-Zwist auf seinem Höhepunkt war, auf den gemeinsamen Bau eines konkurrenzfähigen Passagierflugzeugs einigen konnten, wie die Gründung von Airbus Industrie im Jahre 1970 zeigte. $^{72}$

71. G. BRUNN, Die Europäische Einigung, Reclam, Stuttgart, 2002, S.133.

72. Vgl. W. KRIEGER, Internationale Politik und zivile Luftfahrt. Die historische Bedeutung des Airbus-Konsortiums, in: G. CLEMENS (Hrsg.), Nation und Europa. Studien zum internationalen Staatensystem im 19. und 20. Jahrhundert (Festschrift für Peter Krüger zum 65. Geburtstag), Franz Steiner Verlag, Stuttgart, 2001, S. 251-270. 
